\title{
Increased postoperative day one discharges after implementation of a hysterectomy enhanced recovery pathway: a retrospective cohort study
}

\section{Augmentation du nombre de congés au premier jour après mise en ouvre d'une voie de récupération rapide après hystérectomie: étude rétrospective de cohorte}

\author{
Elizabeth C. Miller, MD • Daniel I. McIsaac, MD • Alan Chaput, MD • \\ Jonathan Antrobus, MD • Hassan Shenassa, MD • Anne Lui, MD
}

Received: 11 March 2014 / Accepted: 13 February 2015/Published online: 28 February 2015

(C) Canadian Anesthesiologists' Society 2015

\begin{abstract}
Purpose In 2011, the hysterectomy enhanced recovery (HER) pathway, a multi-disciplinary, evidence-based care plan designed to improve recovery after open gynecologic surgery for non-malignant lesions, was introduced at The Ottawa Hospital (TOH). This before-and-after study examined the impact of the HER pathway on postoperative day (POD) 1 hospital discharge.

Methods Ethical approval was obtained. This retrospective cohort study included patients who had undergone open abdominal gynecologic surgery for non-
\end{abstract}

This article is accompanied by an editorial. Please see Can J Anesth 2015; 62: this issue.

Author contributions Elizabeth C. Miller was involved with the study design, performed the chart review, and wrote the manuscript. Daniel I. McIsaac was involved with the study design, performed the data analysis, and contributed to writing the manuscript. Jonathan Antrobus and Hassan Shenassa were involved with the hysterectomy enhanced recovery (HER) pathway design and implementation. Alan Chaput was involved with the study design and methodology. Anne Lui was the Senior Author and leader in the development of the HER pathway. She was also involved with the study design. All authors reviewed the final manuscript and contributed to the study.

E. C. Miller, MD · D. I. McIsaac, MD - A. Chaput, MD ·

A. Lui, MD

Department of Anesthesia, The Ottawa Hospital, University of Ottawa, Ottawa, ON, Canada

E. C. Miller, MD ( $\varangle)$

PGY3 Anesthesia, University of Ottawa Department of Anesthesia \& The Ottawa Hospital Research Institute, c/o The Ottawa Hospital, Civic Campus Room B311, 1053 Carling Avenue Mail Stop 249, Ottawa, ON K1Y 4E9, Canada

e-mail: emil1015@uottawa.ca malignant lesions at TOH Civic Campus between July 2010 and September 2012 (the year before and year after HER implementation). Patients were analyzed in either a preHER or post-HER group depending on their surgery date. Patients with chronic pain and emergent surgery were excluded. Data were obtained via medical chart review. Our primary outcome was the percentage of POD 1 discharges before and after HER implementation. Secondary outcomes included return to hospital within 30 days of discharge, median length of stay (LOS), clinician compliance with HER, and an exploratory analysis with multivariable modelling to evaluate which aspects of the HER independently predicted POD 1 discharge. Variables used included American Society of Anesthesiologists physical status ( $\geq I I)$, prior abdominal surgery, body mass index, use of transversus abdominis plane blocks, and anesthetic type.

Results Among the 223 patients, significantly more POD 1 discharges occurred for post-HER compared to pre-HER patients $(34 \%$ vs $7 \%$, respectively; adjusted odds ratio $[O R]=7.33 ; 95 \%$ confidence interval $[C I]=3.05$ to

\footnotetext{
A. Chaput, MD

University of Ottawa Faculty of Medicine Post Graduate Medical

Education, Faculty of Medicine, Ottawa, ON, Canada

J. Antrobus, MD

Borders General Hospital, Roxburghshire, UK

H. Shenassa, MD

Department of Obstetrics \& Gynecology, The Ottawa Hospital,

University of Ottawa, Ottawa, ON, Canada
} 
17.62). Rates of return to hospital at 30 days were similar between the groups (10\% post-HER and $13 \%$ pre-HER; adjusted $O R=0.74 ; 95 \% C I=0.32$ to 1.74$)$. The median length of stay was two days in the post-HER group and three days in the pre-HER group $(P<0.0001)$. Only inhalational general anesthesia was independently associated with decreased odds of POD 1 discharge (adjusted $\mathrm{OR}=0.16,95 \% \mathrm{CI}=0.04$ to 0.65 ).

Conclusion For patients undergoing abdominal hysterectomy, implementation of a HER pathway is associated with a higher POD 1 discharge rate, with no increase in the early return to hospital rate.

\section{Résumé}

Objectif La voie de récupération rapide après hystérectomie (RRAH), un programme de soins multidisciplinaires reposant sur des données probantes et conçu pour améliorer la récupération après chirurgie gynécologique ouverte pour lésion non maligne, a été introduite en 2011 à l'hôpital d'Ottawa (TOH - The Ottawa Hospital). Cette étude avant/après a analysé l'impact de la voie de RRAH sur le congé de l'hôpital au jour postopératoire (POD) 1.

Méthodes Le comité d'éthique de la recherche a donné son accord. Cette étude de cohorte rétrospective a inclus des patientes qui avaient subi une chirurgie gynécologique abdominale ouverte pour des lésions non malignes au TOH Civic Campus entre juillet 2010 et septembre 2012 (l'année précédant et l'année suivant la mise en ouvre de la RRAH). Les patientes ont fait l'objet d'une analyse selon qu'elles entraient dans le groupe pré-RRAH ou post-RRAH, d'après la date de la chirurgie. Les patientes souffrant de douleurs chroniques et celles qui ont été opérées en urgence ont été exclues. Les données ont été extraites par l'étude des dossiers médicaux. Notre critère d'évaluation principal était le pourcentage de congés POD-1 avant et après la mise en cuvre de la RRAH. Les critères d'évaluation secondaires ont inclus le retour à l'hôpital dans les 30 jours suivant le congé, la durée médiane de séjour (DMS), l'observance de la RRAH par le clinicien et une analyse exploratoire avec modélisation multifactorielle pour évaluer quels aspects de la RRAH pouvaient prédire de façon indépendante le congé au POD-1. Les variables ont inclus le statut physique ( $\geq$ II) de l'American Society of Anesthesiologists, les antécédents de chirurgie abdominale, l'indice de masse corporelle, l'utilisation de blocs dans le plan du muscle transverse de l'abdomen et le type d'anesthésique utilisé.

Résultats Parmi les 223 patientes, il y a eu significativement plus de congés au POD-1 dans le groupe post-RRAH que dans le groupe de patientes pré-RRAH (respectivement, $34 \%$ contre $7 \%$; rapport de cotes [RC] ajusté: 7,33; intervalle de confiance [IC] à $95 \%$ : 3,05 à 17,62). Les pourcentages de retours à l'hôpital à 30 jours ont été comparables entre les groupes (10\% post-RRAH et $13 \%$ pré-RRAH; RC ajusté: 0,74; IC à $95 \%=0,32$ à 1,74). La durée médiane de séjour a été de 2 jours pour le groupe post-RRAH et de 3 jours pour le groupe pré-RRAH $(P<0,0001)$. Seule, l'anesthésie générale inhalée a été associée de façon indépendante à une diminution des chances de congé au POD-1 (RC ajusté: 0,16, IC à $95 \%$ : 0,04 à 0,65).

Conclusion Pour les patientes subissant une hystérectomie par voie abdominale, la mise en ouvre d'une voie de RRAH est associée à un taux supérieur de congés au POD-1, sans augmentation de la fréquence de retours précoces à l'hôpital.

Hysterectomy is the most commonly performed nonobstetrical surgery amongst Canadian women. ${ }^{1}$ Average hospital length of stay (LOS) after open hysterectomy is 4.4 days. $^{2}$ Prolonged LOS increases bed utilization, delays operating room (OR) access, and can impede patients from receiving urgent surgery. This problem was of particular concern at our institution because $34 \%$ of open hysterectomies and myomectomies were cancelled at The Ottawa Hospital (TOH) in 2010 owing to a shortage of inpatient beds. Although the less invasive vaginal, laparoscopic, and robot-assisted techniques can accelerate recovery, diminish postoperative pain, ${ }^{3}$ and shorten the LOS, ${ }^{4}$ open techniques are indicated in patients with a large uterus, significant obesity, failed minimally invasive surgery (MIS), ${ }^{2}$ or where a lack of resources limits the use of MIS.

Recognizing the negative impact of cancelling elective surgery and the potential benefit of enhanced recovery, a group of anesthesiologists and gynecologists at $\mathrm{TOH}$ established a multi-disciplinary team to address the issues underlying surgical cancellations. At postoperative followup visits, open hysterectomy and myomectomy patients, their surgeons, and nurses were asked about presumed barriers to discharge from hospital. The top reasons described were pain, nausea and vomiting, ileus, inability to mobilize, and sedation. Even patients experiencing a typical recovery were not routinely discharged until postoperative day (POD) 3. Our multi-disciplinary care team therefore committed to designing and implementing an enhanced recovery pathway for women undergoing open abdominal gynecologic surgery for a non-malignant lesion.

Enhanced recovery or "fast track" programmes optimize perioperative care and may accelerate recovery, reduce morbidity, and shorten the LOS. ${ }^{5,6}$ Enhanced recovery pathways have been efficacious in improving 
outcomes after colorectal and orthopedic surgery, ${ }^{7-10}$ whereas enhanced recovery following gynecologic surgery has been less clearly defined. ${ }^{11}$ The few multifaceted enhanced recovery pathways currently described in the literature enrolled fewer than 100 patients, ${ }^{12,13}$ were specific to minimally invasive procedures, ${ }^{12,14}$ or mixed benign and malignant diagnoses. ${ }^{15,16}$ Some investigations did not compare their pathway to a previous standard of care. $^{17}$

To guide the development of our pathway, we performed a structured review of the literature using PubMed, Google Scholar, and Embase databases to identify evidence-based interventions associated with improved pain control and functional recovery, decreased nausea and vomiting, or decreased LOS after gynecologic surgery. Our review revealed that: 1) spinal anesthesia, in comparison with general anesthesia, is associated with shorter sick leaves ${ }^{18}$ and improved quality of recovery and of life scores; ${ }^{19}$ 2) compared with general anesthesia, spinal anesthesia with intrathecal morphine reduces postoperative pain, ileus, fatigue, hospital LOS, and nausea and vomiting for up to $24 \mathrm{hr}$ postoperatively; ${ }^{20,21}$ 3) transversus abdominis plane (TAP) blocks are associated with improved postoperative pain control and reduced rates of nausea and vomiting; ${ }^{22} 4$ ) foundational multi-modal analgesia achieves postoperative pain control effectively and efficiently. ${ }^{23,24}$

\section{Hysterectomy enhanced recovery pathway}

Review of the literature, in combination with clinical expertise of the multi-disciplinary team was used to create the hysterectomy enhanced recovery (HER) pathway. The HER pathway is a collaborative, multi-disciplinary approach to perioperative patient care that is delineated into preoperative, intraoperative, and postoperative components (Table 1). In our study, all patients who were to undergo open abdominal hysterectomy or myomectomy were identified by their gynecologist during their preoperative visits and were enrolled in the HER pathway. These patients were educated preoperatively about the HER pathway during an anesthesiology consultation and by nurses in the pre-anesthesia unit. Pre-emptive analgesics (acetaminophen $650 \mathrm{mg}$ po and celecoxib 200 or $400 \mathrm{mg}$ po) were prescribed. Preoperative sedatives and opioids were avoided. We chose these analgesics for our pathway to avoid medications with side effects such as nausea and sedation. Anesthesia care was provided by a consistent group of anesthesiologists. Although intraoperative care was ultimately at the discretion of the anesthesiologist, the group was educated and directed to provide spinal anesthesia with $0.75 \%$ hyperbaric bupivacaine (10.0-12.5 mg) and intrathecal morphine $(100 \mu \mathrm{g})$, anesthesiologistadministered ultrasonography-guided TAP blocks with $0.5 \%$ ropivacaine with epinephrine $(5 \mu \mathrm{g} / \mathrm{ml})$, and propofol-based general anesthesia with an advanced airway. Spinal anesthesia was not used as a sole modality. Rather, it was used primarily for analgesia. Patients also received intraoperative dexamethasone (4-8 $\mathrm{mg}$ ) and foundational analgesia postoperatively (including acetaminophen $650 \mathrm{mg}$ po $\mathrm{q} 4 \mathrm{~h}$ and celecoxib 200 or $400 \mathrm{mg}$ po $\mathrm{q} 12 \mathrm{~h}$ ). Providers were asked to avoid volatile anesthetics and intravenous patient-controlled analgesia. All patients also received standard intraoperative gynecological surgical care per $\mathrm{TOH}$ protocol, including the World Health Organization Surgical Safety Checklist, preoperative antibiotic prophylaxis, and a Foley catheter. Postoperatively, patients were provided with oral fluids and a snack. They were assisted with mobilization once they reached the ward. The care team provided orders for removing the Foley catheters and saline-locking intravenous lines once patients had mobilized and were drinking. Patients were discharged when deemed ready by the

Table 1 Hysterectomy enhanced recovery pathway

\begin{tabular}{|c|c|c|}
\hline Preoperative & Intraoperative & Postoperative \\
\hline $\begin{array}{l}\text { - Patient identified by gynecology (all } \\
\text { patients having planned benign open } \\
\text { abdominal procedures) } \\
\text { - Anesthesia consult \& patient education } \\
\text { - Pre-emptive analgesia (acetaminophen } \\
650 \mathrm{mg} \text { po and celecoxib } 200 \text { or } 400 \mathrm{mg} \\
\text { po) before the OR } \\
\text { - Avoidance of preoperative sedatives } \\
\text { - Plan for discharge on POD } 1\end{array}$ & $\begin{array}{l}\text { - SAB with } 0.75 \% \text { hyperbaric bupivacaine }(10- \\
12.5 \mathrm{mg}) \text { and intrathecal epimorph }(100 \mu \mathrm{g}) \\
\text { - Ultrasound-guided TAP blocks administered by } \\
\text { anesthesiology with } 0.5 \% \text { ropivacaine and } \\
5 \mu \mathrm{g} \cdot \mathrm{mL}^{-1} \text { epinephrine } \\
\text { - Propofol infusion for general anesthesia } \\
\text { - PONV prophylaxis (dexamethasone) }\end{array}$ & $\begin{array}{l}\text { - Foundational analgesia (acetaminophen } \\
650 \mathrm{mg} \text { po } \mathrm{q} 4 \mathrm{~h} \text { \& celecoxib } 200 \text { or } \\
400 \mathrm{mg} \text { po } \mathrm{q} 12 \mathrm{~h} \text { ) } \\
\text { - Avoid IV PCA } \\
\text { - Assistance with mobility once on the } \\
\text { Ward } \\
\text { - Remove Foley catheter and saline-lock } \\
\text { IV once patient drinking well } \\
\text { - Discharge home based on order from } \\
\text { gynecology }\end{array}$ \\
\hline
\end{tabular}

$\mathrm{SAB}=$ subarachnoid block; TAP $=$ transversus abdominis plane; IV PCA $=$ intravenous patient-controlled analgesia; $\mathrm{PONV}=$ postoperative nausea and vomiting; POD $1=$ postoperative day 1 
surgical team (i.e., we did not use a standardized set of discharge criteria).

We launched the HER pathway in July 2011 at the Civic Campus of $\mathrm{TOH}$ with the goal of reducing perioperative patient stress, improving quality of recovery, and targeting discharge on POD 1 when appropriate. Nurses in the preoperative assessment unit, recovery room, and on the ward were educated about the HER pathway by motivated nurse educators who were active in developing the pathway. A small group of anesthesiologists were involved in educating the anesthesia group. The components of the pathway were posted in the OR to provide a guideline for the interventions, dosing, and postoperative orders.

We then undertook a retrospective study in patients who had undergone an open hysterectomy or myomectomy for non-malignant disease at the Civic Campus of $\mathrm{TOH}$ one year prior to and one year following implementation of the HER pathway to test our hypothesis that HER pathway implementation would result in an increased POD 1 discharge rate. We concurrently analysed the pathway's impact on hospital readmission and post-discharge emergency room visits, which we hypothesized could be an unintended consequence of early discharge. As a secondary objective, we determined the median LOS. The preoperative and postoperative components of the pathway and intraoperative intravenous dexamethasone were provided to all HER patients. Because compliance with the intraoperative aspects of the HER pathway was variable, we pursued an exploratory analysis specific to the intraoperative aspect of the pathway to determine if certain anesthetic aspects of it were independently associated with POD 1 discharge.

\section{Methods}

After obtaining ethical approval (TOH Research Ethics Board file \#20130039), we undertook a single-centre, retrospective cohort study at $\mathrm{TOH}$, a tertiary care health sciences centre in Ottawa, Ontario, Canada. Requirement for explicit written consent for participation in the pathway was waived.

\section{Primary intervention}

Our primary intervention was introduction of the HER pathway (Table 1).

Cohort and covariates

All adult patients who underwent an open gynecologic operation for non-malignant disease via abdominal incision between July 2010 and September 2012 were identified. Patients with pre-existing chronic pain or who underwent non-elective surgery were excluded. Study dates were chosen to include the year prior to and the year following implementation of the HER pathway. Patients undergoing surgery between July 2010 and June 2011 made up the preHER group, and patients whose surgery took place between July 2011 and September 2012 made up the post-HER group. Data were extracted via manual chart review, and a second reviewer confirmed the data. The data were deidentified to maintain patient confidentiality. Our reporting is in keeping with the Strengthening the Reporting of Observational Studies in Epidemiology statement. ${ }^{25}$ Extracted data included procedure type (hysterectomy, myomectomy, salpingo-oophorectomy), American Society of Anesthesiologists (ASA) physical status, smoking status, history of prior abdominal surgery, body mass index (BMI), preoperative hemoglobin level, anesthesia type (inhalational general anesthesia alone, propofol-based general anesthesia alone, inhalational general anesthesia plus spinal anesthesia, or propofol-based general anesthesia plus spinal anesthesia), provision of a TAP block, date of the procedure, return to the emergency department (ED), and readmission to $\mathrm{TOH}$ within 30 days of discharge. The primary outcome, POD 1 discharge, was obtained for each patient directly from an electronic health records database. For the patients in the post-HER group, we also used written progress notes in the patients' charts to determine reasons for their being discharged later than POD 1.

\section{Outcomes}

The primary outcome was the percentage of patients discharged on POD 1 following benign, open gynecologic surgery before and after implementation of the HER pathway. The study did not assess eligibility for discharge based on specific criteria. Instead, we report the hospital discharge times as documented in the medical record. Our secondary outcomes included 1) a combination of return to the ED or readmission within 30 days of discharge, as a balancing measure, to evaluate whether earlier discharge was associated with an increased rate of early return to hospital; 2) the difference in median LOSs; and 3) clinician compliance with the HER pathway (measured as the percentage of patients undergoing all intraoperative interventions: propofol-based general anesthesia plus spinal anesthesia, TAP block). Finally, because not all of the patients had been exposed to all anesthetic interventions of the intraoperative component of the HER pathway, we used multi-variable modelling in an exploratory analysis to determine if any particular anesthetic intervention was independently associated with POD 1 discharge. 
Analysis

Demographic variables (Table 2) were compared between patients in the pre-HER and post-HER groups using absolute standardized differences. ${ }^{26}$ Although there is no consensus on a standard cutoff, we considered that a difference of $10 \%$ or less denoted a non-substantial difference.

The primary analysis consisted of a Chi square test and multivariable logistic regression to determine if having surgery before or after HER pathway implementation was associated with discharge on POD 1 . The multivariable model included ASA physical status ( $\leq$ II $v s \geq$ III), BMI, age, and procedure type (as a three-level categorical variable). Covariates were chosen a priori based on identified predictors of extended hospital stay. ${ }^{27}$ Our multivariable model had a c-statistic of 0.77 , demonstrating moderate discriminatory performance.

For our secondary analysis, we conducted a Chi square test and multivariate logistic regression, employing the covariates noted above. The unadjusted difference in the median LOS, reported as median (interquartile range), between groups was evaluated using a Wilcoxon test.

We also measured compliance with the intraoperative aspects of the HER pathway. The HER anesthetic patients were defined as patients who received all three of the intraoperative anesthetic components of the HER pathway: propofol-based general anesthesia plus spinal anesthesia and an anesthesiologist-administered ultrasonographyguided TAP block. Partial HER anesthetic patients received two of the three anesthetic components of the HER pathway. Non-HER anesthetic patients were given inhaled general anesthesia with neither spinal anesthesia nor a TAP block.

Our exploratory analysis was undertaken to evaluate independent intraoperative predictors of POD 1 discharge in hysterectomy and myomectomy patients (those with salpingo-oophorectomy were excluded because only three were done). Fisher's exact test or Student's $t$ tests were used to assess the unadjusted strength of association between the following factors and POD 1 discharge: 1) anesthesia type (as a three-level categorical variable: propofol-based general anesthesia plus spinal anesthesia as reference $v s$ inhalational general anesthesia only or inhalational general anesthesia plus spinal anesthesia); 2) provision of a TAP block; 3) surgical factors; 4) demographic factors; 5) temporal effects (first or second $50 \%$ of patients treated with the HER pathway) (Table 4). We chose an a priori level of significance of 0.1 to determine inclusion in a logistic regression model to determine the impact of the different anesthetic types and TAP block provision on POD 1 discharge independent of strongly predictive demographic factors. Because only one post-HER patient had propofol-based general anesthesia
Table 2 Patient demographics

\begin{tabular}{|c|c|c|c|}
\hline & $\begin{array}{l}\text { pre-HER } \\
n=100\end{array}$ & $\begin{array}{l}\text { post-HER } \\
n=123\end{array}$ & $\begin{array}{l}\text { Absolute } \\
\text { standardized } \\
\text { difference }(\%)\end{array}$ \\
\hline Age & $45(9)$ & $45(9)$ & 0 \\
\hline \multicolumn{4}{|l|}{ Mean (SD) } \\
\hline $\begin{array}{l}\mathrm{ASA} \leq \mathrm{II} \\
n(\%)\end{array}$ & $74(74.0)$ & $95(77.0)$ & 7 \\
\hline $\begin{array}{l}\text { BMI }\left(\mathrm{kg} \cdot \mathrm{m}^{-2}\right) \\
\text { Mean }(\mathrm{SD})\end{array}$ & $28.2(5.6)$ & $28.6(6.2)$ & 1.1 \\
\hline $\begin{array}{l}\text { Ever smoker } \\
n(\%)\end{array}$ & $33(33.0)$ & $36(29.3)$ & -8.7 \\
\hline $\begin{array}{l}\text { Prior abdominal surgery } \\
n(\%)\end{array}$ & $65(66.3)$ & $83(67.5)$ & 4.3 \\
\hline $\begin{array}{l}\text { Preoperative } \mathrm{Hg} \\
\text { Mean (SD) }\end{array}$ & $128(18)$ & $128(18)$ & 0 \\
\hline Surgery type & & & \\
\hline $\begin{array}{l}\text { Hysterectomy } \\
n(\%)\end{array}$ & $72(72.0)$ & $92(74.8)$ & 6.8 \\
\hline $\begin{array}{l}\text { Myomectomy } \\
n(\%)\end{array}$ & $21(21.0)$ & $28(22.8)$ & 4.8 \\
\hline $\begin{array}{l}\text { Ovarian/Adnexal } \\
n(\%)\end{array}$ & $7(7.0)$ & $3(2.4)$ & -24.3 \\
\hline Anesthetic $*, * *$ & & & \\
\hline $\begin{array}{l}\text { Inhaled general anesthesia } \\
\text { only } \\
n(\%)\end{array}$ & $81(81.0)$ & $44(35.8)$ & -102 \\
\hline $\begin{array}{l}\text { Propofol based general } \\
\text { anesthesia only } \\
n(\%)\end{array}$ & $0(0.0)$ & $1(0.8)$ & 14 \\
\hline $\begin{array}{l}\text { Inhaled general anesthesia } \\
\text { plus spinal } \\
n(\%)\end{array}$ & $18(18.0)$ & $21(17.1)$ & 3 \\
\hline $\begin{array}{l}\text { Propofol based general } \\
\text { anesthesia plus spinal } \\
n(\%)\end{array}$ & $1(1.0)$ & 55 (44.7) & 122 \\
\hline $\begin{array}{l}\text { Transversus abdominis } \\
\text { plane (TAP) block } \\
n(\%)\end{array}$ & $18(18.0)$ & $69(56.0)$ & 85.6 \\
\hline
\end{tabular}

HER = hysterectomy enhanced recovery; ASA = American Society of Anesthesiologist; $\mathrm{BMI}=$ body mass index; $\mathrm{Hg}=$ hemoglobin; $\mathrm{MAC}=$ monitored anesthesia care

*Anesthesia for pre-HER and post-HER consisted of a combination of: inhaled general anesthesia, propofol anesthesia, spinal for analgesia, and TAP blocks. Spinal anesthesia was not used as a sole modality; patients received either inhaled general anesthesia or propofol anesthesia, with or without spinal analgesia. The number (and percent) of patients who additionally received TAP blocks is shown in the last row. **Anesthetic type for two patients is missing from the post-HER group

alone, her data was excluded from the exploratory analysis. All analyses were performed in SAS v 9.4 for Windows (SAS Institute, Cary, NC, USA). 


\section{Results}

A total of 257 patients were identified as having undergone open gynecologic surgery for non-malignant disease via abdominal incision between July 2010 and September 2012. We reviewed their charts. Amongst them, 113 underwent surgery during the year prior to HER implementation in July 2011 (pre-HER), and 144 underwent surgery following HER implementation (post-HER). Overall, 13 pre-HER patients and 21 post-HER patients were excluded because they had had a diagnosis of chronic pain or had undergone non-elective surgery. We were then left with 100 patients for analysis in the pre-HER group and 123 patients for analysis in the post-HER group (Fig. 1).

Patient demographics and anesthetic techniques

The patient characteristics in the two groups were similar (Table 2). Hysterectomy was the most common surgical procedure followed by myomectomy. Ovarian and adnexal surgeries were rarely performed.

Anesthesia pre-HER and post-HER consisted of inhalational general anesthesia alone, propofol-based general anesthesia alone, inhalational general anesthesia plus spinal anesthesia, or propofol-based general anesthesia plus spinal anesthesia (Table 2). No patient received spinal

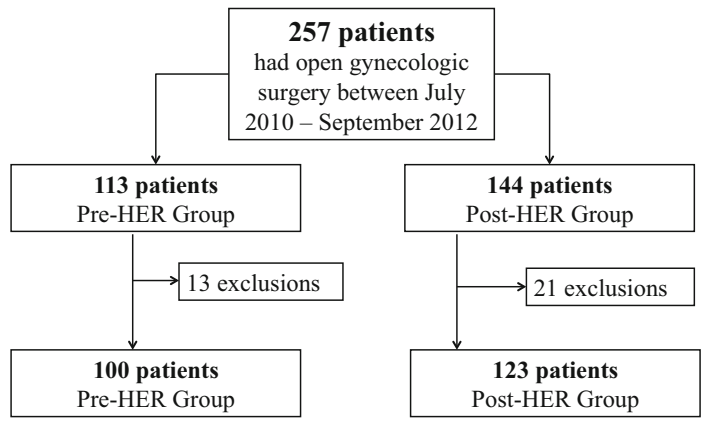

Fig. 1 Patient flow diagram anesthesia as a sole modality. It was administered primarily for analgesia. All patients were given either inhaled general anesthesia or propofol-based general anesthesia as well. The number (and percent) of patients who additionally underwent a TAP block is shown in Table 2. There were notable differences in regard to anesthetic techniques preHER $v s$ post-HER implementation. In the pre-HER group, $81 \%$ of patients were given inhaled general anesthesia only, $18 \%$ were given inhaled general anesthesia plus spinal anesthesia, and only $1 \%$ were given propofol-based general anesthesia plus spinal anesthesia. Following launch of the HER pathway, $35.8 \%$ were given inhaled general anesthesia only, $17.1 \%$ were given inhaled general anesthesia plus spinal anesthesia, and $44.7 \%$ were given propofol-based general anesthesia plus spinal anesthesia. Overall, $18 \%$ of pre-HER patients were subjected to a concurrent TAP block compared with $56 \%$ of the postHER patients. Only one patient (in the post-HER group) was administered propofol-based general anesthesia alone.

\section{Patient outcomes}

A higher percentage of patients in the post-HER group were discharged on POD 1 (our primary outcome) than in the pre-HER group (34\% vs 7\%, respectively; adjusted odds ratio $[\mathrm{OR}]=7.33 ; 95 \%$ confidence interval $[\mathrm{CI}]=3.05$ to 17.62$) \quad($ Table 3$)$. In contrast, the combined rate of early readmission to hospital and visits to the ED (secondary outcomes) (Table 3) were similar in the two groups $(10 \%$ vs $13 \%$ [post-HER vs pre-HER respectively]; adjusted $\mathrm{OR}=0.74 ; 95 \% \mathrm{CI}=0.32$ to 1.74). The median LOS (Table 3) was 2 (1.0-3.0) days in the post-HER group compared to 3 (2.5-3.0) days in the pre-HER group $(P<0.0001)$. Among the patients in the post-HER group, $38 \%$ of patients were described as ready for discharge on POD 1, but only $34 \%$ were actually discharged. Thus, $62 \%$ of the post-HER patients were not deemed ready for discharge on POD 1 because of surgical reasons $(33 \%)$, reason not stated $(32 \%)$, pain $(17 \%)$, nausea $(11 \%)$, urinary retention $(7 \%)$. No benign gynecological

Table 3 Primary and secondary outcomes

\begin{tabular}{|c|c|c|c|c|c|c|}
\hline & $\begin{array}{l}\text { pre-HER } \\
n=100\end{array}$ & $\begin{array}{l}\text { post-HER } \\
n=123\end{array}$ & $\begin{array}{l}\text { Crude } \\
\text { OR }(95 \% \text { CI })\end{array}$ & $P$ value & $\begin{array}{l}\text { Adjusted OR } \\
(95 \% \mathrm{CI})\end{array}$ & $P$ value \\
\hline $\begin{array}{l}\text { POD } 1 \text { discharges } \\
n(\%)\end{array}$ & $7(7.0)$ & $42(34.0)$ & 6.89 (2.93 to 16.18$)$ & $<0.001$ & 7.33 (3.05 to 17.62 ) & $<0.001$ \\
\hline $\begin{array}{l}\text { Readmission or ER visit within } 30 \text { days of } \\
\quad \text { discharge } \\
n(\%)\end{array}$ & $13(13.0)$ & $12(10.0)$ & $0.72(0.31$ to 1.66$)$ & 0.45 & $0.74(0.32$ to 1.74$)$ & 0.49 \\
\hline $\begin{array}{l}\text { Length of stay } \\
\text { median (IQR) }\end{array}$ & $3(2.5-3.0)$ & $2(1.0-3.0)$ & & $<0.0001$ & & \\
\hline
\end{tabular}

$\mathrm{HER}=$ hysterectomy enhanced recovery; $\mathrm{OR}=$ odds ratio; $\mathrm{CI}=$ confidence interval; $\mathrm{ER}=$ emergency room; IQR $=$ interquartile range 


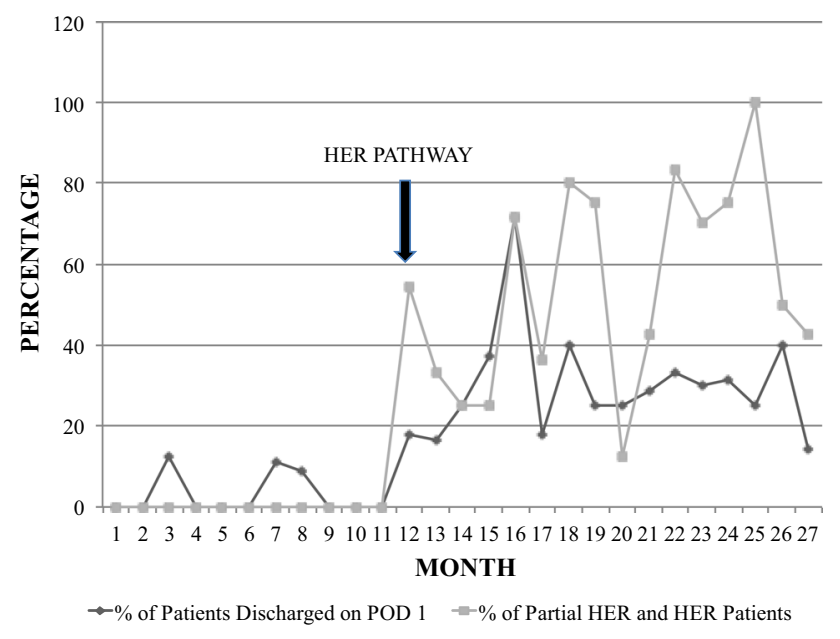

Fig. 2 Compliance with the anesthetic interventions of the hysterectomy enhanced recovery (HER) pathway. $\mathrm{POD}=$ postoperative day

surgeries were cancelled at $\mathrm{TOH}$ following implementation of the HER pathway because of bed unavailability.

Regarding compliance with the HER pathway, all patients were provided with preoperative education, foundational analgesia, and intravenous dexamethasone. In the post-HER group, $35 \%$ of patients received all three intraoperative interventions, $36 \%$ received two of the three, and $29 \%$ received one or none. Compliance with the HER anesthesia protocol relative to the percentage of POD 1 discharges are graphed in Fig. 2.

In our exploratory analysis (Table 4), inhaled general anesthesia only was found to be associated with decreased odds of POD 1 discharge in this unadjusted analysis (unadjusted $\mathrm{OR}=0.08 ; 95 \% \mathrm{CI}=0.03$ to 0.27 ). Patients given inhaled general anesthesia plus spinal anesthesia had a similar likelihood of being discharged on POD 1 as those given propofol-based general anesthesia plus spinal anesthesia, (unadjusted $\mathrm{OR}=0.51 ; 95 \% \mathrm{CI}=0.18$ to 1.43). Inhaled general anesthesia was independently associated with a decreased odds of POD 1 discharge (adjusted $\mathrm{OR}=0.16 ; 95 \% \mathrm{CI}=0.04$ to $0.65 ; P=0.01$ ).

\section{Discussion}

Implementation of the HER pathway in July 2011 was associated with higher rates of POD 1 discharge, with no increase in the rate of early return to hospital compared to that for the year prior to program implementation. This supports early discharge being appropriate following implementation of the HER pathway. Furthermore, There was a meaningful reduction (one day per admission) in the median hospital LOS after implementation of the HER pathway.
The HER pathway is a multi-disciplinary and multifaceted intervention that spans only the perioperative period. Hence, we were not able to determine if the increased POD 1 discharge rate was attributable to specific aspects of the pathway. Compliance with the preoperative and postoperative portions of the pathway was excellent per our measures, although significant variability existed in the application of the intraoperative component. This variability allowed an exploratory analysis of the impact of the intraoperative interventions. For example, nausea and pain had been identified during program development as barriers to discharge. It is not surprising, then, that avoidance of inhaled general anesthesia, which may contribute to nausea and vomiting, ${ }^{28}$ was associated with increased POD 1 discharge. It is likely that the implementation of this multi-faceted pathway caused a change in hospital culture, perceptions, and patient care goals that also contributed to our improved record of early discharge. Despite variations in the application of intraoperative interventions, we were able to discharge at least $20 \%$ of patients on POD 1 during 13 of the 15 months after HER implementation, a proportion that was never achieved before implementing the HER pathway.

Of particular interest to physicians and hospital administrators is that our pathway required no change in hospital policy, additional funding, or personnel. No new resources were required to implement it. A team of nurses and anesthesiologists who were already employed at the hospital educated the perioperative team and the patients about the pathway. Education was provided via preexisting modalities, such as grand rounds and nurse educators for the staff and nurses in the pre-anesthetic unit for the patients. The specific anesthetic interventions of the HER pathway are not novel. They are all standard options for routine anesthetic care. For our pathway, they were combined to form a multi-pronged care plan to optimize analgesia usage and patient recovery. Increased OR efficiency may be realized through placement of TAP blocks preoperatively in a "block room" or postoperatively in the postoperative anesthesia care unit.

Our study makes a unique contribution to the literature and features several strengths. Implementation of an enhanced recovery pathway at our institution readied patients for a discharge on POD 1, which is earlier than other studies of gynecological patients. ${ }^{16-19}$ Studies of enhanced recovery in gynecology have focused on laparoscopic surgery or featured a mix of benign and malignant surgical indications. ${ }^{15,16} \mathrm{We}$ describe a pathway oriented specifically at an open surgical approach for treating benign disease that allows practitioners to identify patients appropriate for HER pathway application. Over the study period, the only institutional change at TOH was implementation of the HER pathway. The surgeons and 
Table 4 Exploratory analysis of post-HER group

\begin{tabular}{|c|c|c|c|c|c|c|}
\hline \multirow[t]{2}{*}{ Predictors } & \multirow{2}{*}{$\begin{array}{l}\text { Discharge } \\
\text { on POD } 1\end{array}$} & \multirow{2}{*}{$\begin{array}{l}\text { Discharge } \\
\text { after } \\
\text { POD } 1\end{array}$} & \multicolumn{2}{|l|}{ Univariable analysis } & \multicolumn{2}{|c|}{ Multivariable analysis } \\
\hline & & & $\begin{array}{l}\text { Crude } \\
\text { OR }(95 \% \mathrm{CI})\end{array}$ & $P$ value & $\begin{array}{l}\text { Adjusted } \\
\text { OR }(95 \% \mathrm{CI})\end{array}$ & $P$ value \\
\hline $\begin{array}{l}\text { Propofol based general anesthesia plus spinal }{ }^{\dagger} \\
n(\%)\end{array}$ & $30(55)$ & $25(45)$ & 1 & reference & 1 & reference \\
\hline $\begin{array}{l}\text { Inhalational general anesthesia plus spinal }{ }^{\dagger} \\
n(\%)\end{array}$ & $13(62)$ & $8(38)$ & $0.51(0.18$ to 1.43$)$ & 0.20 & $0.75(0.24$ to 2.41$)$ & 0.63 \\
\hline $\begin{array}{l}\text { Inhalational general anesthesia only }{ }^{\dagger} \\
n(\%)\end{array}$ & $40(91)$ & $4(9)$ & 0.08 (0.03 to 0.27$)$ & $<0.001$ & $0.16(0.04$ to 0.65$)$ & 0.01 \\
\hline $\begin{array}{l}\text { Transversus abdominis plane } \text { block }^{\dagger} \\
n(\%)\end{array}$ & $33(48)$ & $36(52)$ & $4.58(1.94$ to 10.81$)$ & $<0.001$ & $1.89(0.64$ to 5.63$)$ & 0.25 \\
\hline $\begin{array}{l}\text { First } 50 \% \text { of patients to have surgery after HER } \\
\text { implementation* } \\
n(\%)\end{array}$ & $23(37)$ & $19(31)$ & $0.73(0.35$ to 1.54$)$ & 0.11 & - & - \\
\hline $\begin{array}{l}\text { Ever smoker } \\
n(\%)\end{array}$ & $14(39)$ & $22(61)$ & $1.34(0.60$ to 3.00$)$ & 0.53 & - & - \\
\hline $\begin{array}{l}\text { Body mass index }\left(\mathrm{kg} / \mathrm{m}^{-2}\right)^{\dagger} \\
\text { Mean }\end{array}$ & 26.8 & 28.8 & $0.94(0.88$ to 1.00$)$ & 0.06 & $0.97(0.89$ to 1.05$)$ & 0.41 \\
\hline $\begin{array}{l}\text { Age } \\
\text { Mean }\end{array}$ & 44.9 & 45.1 & $1(0.96$ to 1.05$)$ & 0.95 & - & - \\
\hline $\begin{array}{l}\text { Prior abdominal surgery }{ }^{\dagger} \\
n(\%)\end{array}$ & $33(40)$ & $50(60)$ & 2.27 (0.96 to 5.39$)$ & 0.07 & $2.47(0.94$ to 6.50$)$ & 0.07 \\
\hline $\begin{array}{l}\mathrm{ASA} \geq \mathrm{II}^{\dagger} \\
n(\%)\end{array}$ & $5(17)$ & $23(83)$ & $0.34(0.12$ to 0.98$)$ & 0.04 & $0.68(0.19$ to 2.40$)$ & 0.55 \\
\hline $\begin{array}{l}\text { Total abdominal hysterectomy procedure }{ }^{* *} \\
n(\%)\end{array}$ & $29(32)$ & $63(68)$ & $0.53(0.22$ to 1.23$)$ & 0.15 & - & - \\
\hline
\end{tabular}

anesthesiologists were the same during both the pre-HER and post-HER periods, and no new surgical pathways or initiatives aimed at decreasing LOS were introduced other than the HER pathway. Also, the characteristics of the preHER and post-HER groups were comparable. These features support the contention that the effects described in our report are largely attributable to implementation of the HER pathway. As the data originated from a large, tertiary care, publicly funded centre in Ontario, our findings may be generalizable to similar centres with similar patient populations.

There are several limitations of this study. As a retrospective observational study, it was possible only to control for measured confounders. The surgeon is a potentially important confounder that was not controlled. However, a small group of surgeons at our centre performed all of the surgery described herein, and there were no changes in surgical staff between the pre-HER and post-HER periods. We were not able to measure fully the preoperative aspects of the HER pathway, such as the quality and saturation of patient education, or the spinal or TAP block dosing. We also could not precisely describe the success rates. As a retrospective chart review, there is potential for bias as our data extractor was not blinded. However, data for our primary outcome of interest (POD 1 discharge) were not extracted manually but were provided for each patient directly from an electronic health records database maintained by the hospital. The demographic variables and anesthetic interventions were collected from our computer-generated anesthesia records, which have minimal room for interpretation. To minimise bias, two individuals extracted data for variables that left some room for interpretation (e.g., reason for discharge). There was no washout period between the pre-HER period and 
implementation of the HER pathway, which could have diluted the post-HER data. Although the increase in POD 1 discharges did not increase the rate of return to hospital, a prospective study would be required to conclude that the HER protocol is not associated with other adverse postdischarge outcomes. Also, our data collection was limited to post-discharge returns to our own hospital.

Our results are important from both patient care and quality improvement perspectives. Reducing the LOS to one day could produce system efficiencies and potentially decrease the risk of hospital-acquired complications. Further research is needed to elucidate these hypotheses. It was impossible in our study to determine whether a single element of the pathway-the anesthetic intervention, patient education, changes in nursing practise, planning for discharge on POD 1-was solely responsible for the higher rates of POD 1 discharge. Our results, however, suggest that there is potential for open hysterectomy to become an overnight-stay procedure on a routine basis in selected patients. This could be achieved through implementation of simple changes without additional resources or changes in policy.

Further research and refinement of the HER pathway could produce even greater increases in POD 1 discharge. Assessment of barriers and facilitators of pathway implementation, particularly intraoperative aspects, might identify factors that could improve adherence to applying all three anesthetic interventions, particularly as $53 \%$ of patients still received an inhaled general anesthetic. Prospective study of the HER pathway, including patient centred outcomes (e.g., pain, functional recovery, return to work) could enhance our understanding of the needs of this surgical population. It is possible that enhanced recovery pathways could be applied to gynecologic oncology surgery, including procedures that require laparotomy. ${ }^{29,30}$ Finally, we did not compare the costs of the various interventions used in the pathway or whether there were cost savings with reduced LOS or increased costs due to higher patient flow. These comparisons would be a fascinating area for future research.

In conclusion, implementation of the HER pathway for open non-malignant gynecologic surgery was associated with an increased number of patients discharged on POD 1, without augmenting the number who made an early return to hospital.

Acknowledgement We sincerely thank Dr. Gregory Bryson for his advice and guidance regarding the design of this study.

Funding Funding was provided by the University of Ottawa Department of Anesthesiology.

Competing interests None declared.

\section{References}

1. Canadian Institute for Health Information. "Hysterectomies and Disparities." Health Indicators 2010. Available from URL: https:// secure.cihi.ca/free_products/Healthindicators2010_en.pdf2010. Available at: www.cihi.ca. Search "Hysterectomy" to access "Health Care in Canada 2010" PDF document: P31 (accessed November 2014).

2. Toma A, Hopman WM, Gorwill RH. Hysterectomy at a Canadian tertiary care facility: results of a one year retrospective review. BMC Womens Health 2004; 4: 10.

3. Johnson N, Barlow D, Lethaby A, Tavender E, Curr L, Garry R. Methods of hysterectomy: systematic review and meta-analysis of randomized controlled trials. BMJ 2005; 330: 1478.

4. Reza M, Maeso S, Blasco JA, Andradas E. Meta-analysis of observational studies on the safety and effectiveness of robotic gynecological surgery. Br J Surg 2010; 97: 1772-83.

5. Feldman LS, Lee L, Fiore J Jr. What outcomes are important in the assessment of Enhanced Recovery After Surgery (ERAS) pathways? Can J Anesth 2015; DOI:10.1007/s12630-014-0263-1.

6. Kehlet $H$. Enhanced Recovery After Surgery (ERAS): good for now, but what about the future? Can J Anesth 2015; DOI:10. 1007/s12630-014-0261-1.

7. Wind J, Polle SW, Fung Kon Jin PH, et al. Laparoscopy and/or Fast Track Multimodal Management Versus Standard Care (LAFA) Study Group; Enhanced Recovery after Surgery (ERAS) Group. Systematic review of enhanced recovery programmes in colonic surgery. Br J Surg 2006; 93: 800-9.

8. Greco M, Giovanni C, Beretta L, Gemma M, Pecorelli N, Braga M. Enhanced recovery program in colorectal surgery: a meta-analysis of randomized controlled trials. World J Surg 2014; 38: 1531-41.

9. Varadhan $K K$, Neal KR, Dejong $C H$, Fearon $K C$, Ljungqvist $O$, Lobo $D N$. The enhanced recovery after surgery (ERAS) pathway for patients undergoing major elective open colorectal surgery: a meta-analysis of randomized controlled trials. Clin Nutr 2010; 29: 434-40.

10. Jones EL, Wainwright TW, Foster JD, Smith JR, Middleton RG, Francis NK. A systematic review of patient reported outcomes and patient experience in enhanced recovery after orthopaedic surgery. Ann R Coll Surg Engl 2014; 96: 89-94.

11. Borendal Wodlin N, Nilsson L. The development of fast-track principles in gynecological surgery. Acta Obstet Gynecol Scand 2013; 92: 17-27.

12. Yoong W, Sivashanmugarajan V, Relph S, et al.; Enhanced Recovery After Surgery (ERAS) Team for Gynaecology and Anaesthesia. Can enhanced recovery pathways improve outcomes for vaginal hysterectomy? Cohort control study. J Minim Invasive Gynecol 2014; 21: 83-9.

13. Kroon UB, Radstrom $M$, Hjelthe $C$, Dahlin $C$, Kroon L. Fast-track hysterectomy: a randomized, controlled study. Eur J Obstet Gynecol Reprod Biol 2010; 151: 203-7.

14. Moller C, Kehlet H, Friland SG, Schouenbourg LO, Lund C, Ottesen B. Fast track hysterectomy. Eur J Obstet Gynecol Reprod Biol 2001; 98: 18-22.

15. Carter J, Philp S, Arora $V$. Early discharge after major gynaecological surgery: advantages of fast track surgery. Open J Obstet Gynecol 2011; 1: 1-5.

16. Wijk L, Franzen $K$, Ljungqvist $O$, Nilsson $K$. Implementing a structured enhanced recovery after surgery (ERAS) protocol reduces length of stay after abdominal hysterectomy. Acta Obstet Gynecol Scand 2014; 93: 749-56.

17. Borendal Wodlin N, Nilsson L, Kjolhede P, GASPI study group. The impact of mode of anesthesia on postoperative recovery from fast-track hysterectomy. BJOG 2011; 118: 299-308. 
18. Wodlin NB, Nilsson L, Kjolhede P. Health-related quality of life and postoperative recovery in fast-track hysterectomy. Acta Obstet Gynecol Scand 2011; 90: 362-8.

19. Catro-Alves LJ, De Azevedo VL. De Freitas Braga TF, Goncalves AC, De Oliveira GS Jr. The effect of neuraxial versus general anesthesia techniques on postoperative quality of recovery and analgesia after abdominal hysterectomy: a prospective, randomized, controlled trial. Anesth Analg 2011; 113: 1480-6.

20. Massicotte L, Chalaoui KD, Beaulieu D, Roy JD, Bissonnette F. Comparison of spinal anesthesia with general anesthesia on morphine requirement after abdominal hysterectomy. Acta Anaesthesiol Scand 2009; 53: 641-7.

21. Wodlin NB, Nilsson L, Arestedt K, Kjolhede P; GASPI Study Group. Mode of anesthesia and postoperative symptoms following abdominal hysterectomy in a fast-track setting. Acta Obstet Gynecol Scand 2011; 90: 369-79.

22. Carney J, McDonnell JG, Ochana A, Bhinder R, Laffey JG. The transversus abdominis plane block provides effective postoperative analgesia in patients undergoing total abdominal hysterectomy. Anesth Analg 2008; 107: 2056-60.

23. Moote CA. The prevention of postoperative pain. Can J Anaesth 1994; 41: 527-33.

24. Tan M, Law LS, Gan TJ. Optimizing pain management to facilitate Enhanced Recovery After Surgery pathways. Can J Anesth 2015; DOI:10.1007/s12630-014-0275-x.
25. von Elm E, Altman DG, Egger M, Pocock SJ, Gotzsche PC, Vandenbroucke JP; STROBE Initiative. The Strengthening the Reporting of Observational Studies in Epidemiology (STROBE) statement: guidelines for reporting observational studies. PLoS Med 2007; 4: e296.

26. Austin $P C$. Using the standardized difference to compare the prevalence of a binary variable between two groups in observational research. Commun Stat Simul Comput 2009; 38: 1228-34.

27. Victory $R$, Carey MS, Stitt L. Predictors of length of stay for inpatients having benign gynaecological surgery. J Obstet Gynaecol Can 2005; 27: 43-50.

28. Sneyd JR, Carr A, Byrom WD, Bilski AJ. A meta-analysis of nausea and vomiting following maintenance of anaesthesia with propofol or inhalational agents. Eur J Anaesthesiol 1998; 15: 43345.

29. Letton $C$, Cheung $C$, Nordin A. Does an enhanced recovery integrated care pathway (ICP) encourage adherence to prescribing guidelines, accelerate postoperative recovery and reduce length of stay for gynaecological oncology patients? J Obstet Gynaecol 2013; 33: 296-7.

30. Kalogera E, Bakkum-Gamez JN, Jankowski CJ, et al. Enhanced recovery in gynecologic surgery. Obstet Gynecol 2013; 122(2 Pt 1): $319-28$. 